\title{
t tbar b bbar hadroproduction at NLO accuracy matched with parton shower
}

\author{
Maria Vittoria GARZELLI, Adam KARDOS and Zoltán TRÓCSÁNYI*† \\ Laboratory for Astroparticle Physics, University of Nova Gorica, SI-5000 Nova Gorica, Slovenia \\ INFN, Sezione di Milano-Bicocca, I 20126 Milano, Italy \\ Institute of Physics, University of Debrecen, H-4010 Debrecen P.O.Box 105, Hungary \\ E-mail: Z.Trocsanyi@atomki.hu
}

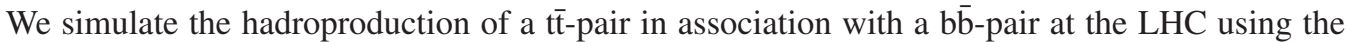
PowHel program. The generated events are stored according to the Les-Houches event format. We interface those events to the PYTHIA shower Monte Carlo program, allowing for decays of massive particles, showering and hadronization, thus leading to predictions for differential distributions at the hadron level that are accurate to next-to-leading order. This is a part of a bigger project in which we have provided similar events for the following final states: (i) $t+\bar{t}$, (ii) $\mathrm{t}+\overline{\mathrm{t}}+H / Z / W$, (iii) $\mathrm{t}+\overline{\mathrm{t}}+\mathrm{b}+\overline{\mathrm{b}}$, (iv) $\mathrm{t}+\overline{\mathrm{t}}+$ jet.
\end{abstract}

The European Physical Society Conference on High Energy Physics

18-24 July, 2013

Stockholm, Sweden

* Speaker.

$\dagger$ This research was supported by the LHCPhenoNet network PITN-GA-2010-264564, Hungarian Scientific Research Fund grant K-101482, the TAMOP-4.2.2.C-11/1/KONV-2012-0010 and TAMOP 4.2.2/B-10/1-2010-0024 projects. 
After the discovery of a Higgs-particle at the LHC [1,2], measuring its couplings to the particles of the Standard Model is a prime task for the experiments. In this respect, the t-quark, being the heaviest known elementary particle plays an outstanding role. According to a recent measurement, the t-quark mass is $m_{\mathrm{t}}=173.5 \pm 0.6 \pm 0.8 \mathrm{GeV}$ [3], indicating a Yukawa coupling of the t-quark $y_{\mathrm{t}}=0.997 \pm 0.008$. Measuring this coupling directly is important also because deviation from the SM expectation could provide signal of physics beyond the SM.

A Higgs-boson of mass $125 \mathrm{GeV}$ cannot decay into a t $\bar{t}$-pair. Thus to measure the $\mathrm{t} \overline{\mathrm{t}} H$ coupling in a model independent way, one should study the hadroproduction of the Higgs-boson in associa-

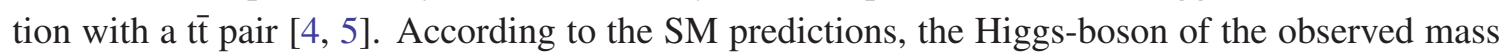

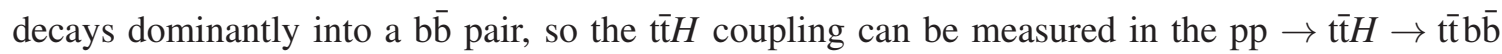
process. This process, however, has an overwhelming background from the direct QCD process pp $\rightarrow \mathrm{t} \overline{\mathrm{t}} \mathrm{b} \overline{\mathrm{b}}$. In order to optimize the experimental selection of the $\bar{t} t H$ events, one needs simulation of the QCD background with high precision.

State of the art theoretical predictions for this background are available at the next-to-leading order (NLO) accuracy $[6,7,8]$. While the NLO corrections are crucial to obtain useful predictions, from the experimental point of view it is also important to estimate correctly the effects of parton showering and hadronization. This can be achieved by the matching of NLO QCD predictions to shower Monte Carlo programs (SMC). We use the POWHEG method for such a matching [9, 10] as implemented in the POWHEG-BOX [11]. In the previous EPSHEP conference we reported [12] about the POwHel framework that combines the POWHEG-BOX and the HELAC-NLO package [13] to simulate the hadroproduction of tit-pairs in association of other hard SM particles. The output of PowHel is simulated events stored according to the Les Houches accord [14] (LHE) that can be fed directly into SMC's to provide events at the hadron level. Using this framework we already provided events for several processes at the $\operatorname{LHC}[15,16,17,18,19,20,21]$.

The technical details of our computations can be found in Ref. [21], where we concluded that the LHE's (pre-showered events) reproduce the distributions at NLO accuracy within the expected precision. To illustrate the level of agreement, we present distributions computed at NLO accuracy as well as from the generated events for (a) the transverse momentum and (b) rapidity of the hardest $b$-jet in Fig. 1. To select events, first we clustered tracks into jets with the $k_{\perp}$-algorithm (as implemented in Fast Jet Ref. [22]) using $R=0.4$. A track was considered a possible jet constituent if $\left|\eta^{\text {track }}\right|<5$. We excluded t-quarks from the set of possible tracks. Then we employed the following selection cuts for these distributions:

1. events with invariant mass of the $b \bar{b}$-jet pair below $m_{b \bar{b}}^{\min }=100 \mathrm{GeV}$ were discarded,

2. $p_{\perp, j}^{\min }=20 \mathrm{GeV}$,

3. we required at least two jets, one $b$ - and one $\bar{b}$-jet, with $\left|y_{b(\bar{b})}\right|<2.5$.

We compare predictions at NLO accuracy and also from LHE's, both generated by PowHel, obtained using fixed and equal renormalization and factorization scales $\mu_{\mathrm{R}}=\mu_{\mathrm{F}}=\mu_{0}=m_{\mathrm{t}}$. (Our NLO predictions agree with those of Ref. [7].) We find that for this fixed scale, the two predictions agree within the scale uncertainties (about $30 \%$ ), although those from the LHE's are about $25 \%$ larger. This difference is rooted in the large NLO corrections (about $80 \%$ for the fixed scale). While the distributions from LHE's also have formal NLO accuracy, these differ from the NLO predictions 

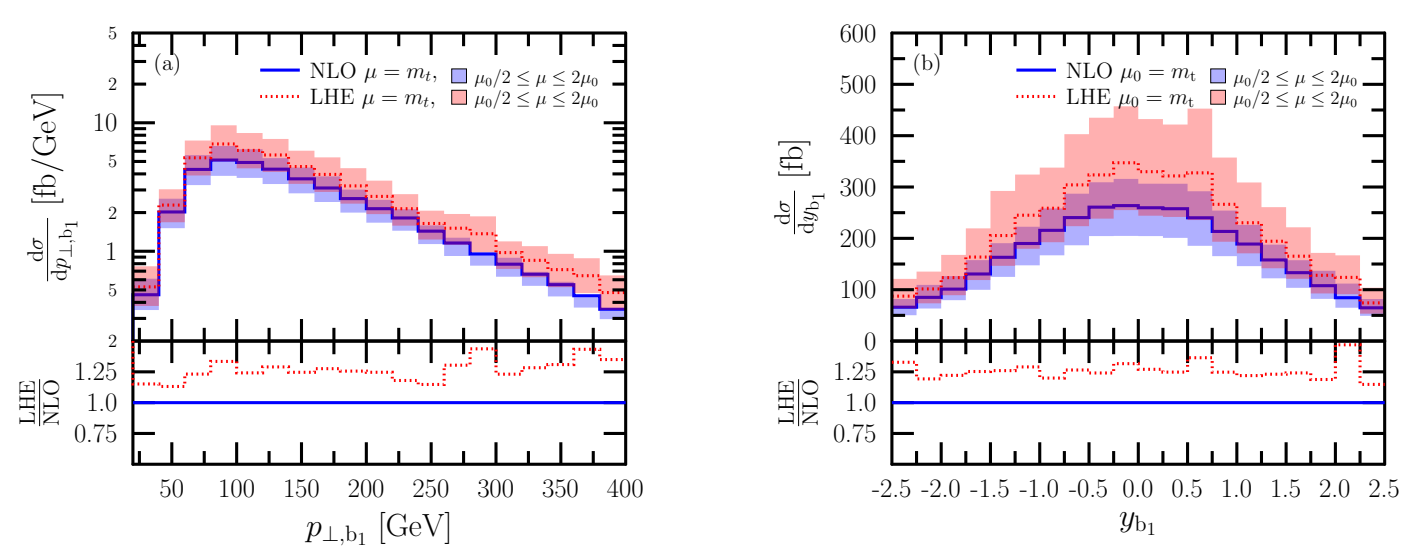

Figure 1: Kinematic distributions of the hardest $b$-jet: (a) transverse momentum, (b) rapidity. Distributions from LHE's are denoted LHE, while those at NLO accuracy by NLO. The shaded band correspond to cross sections obtained from LHE's by varying the (equal) renormalization nad factorization scales around the default one in the range $\left[\mu_{0} / 2,2 \mu_{0}\right]$. The lower panels show the predictions from LHE's normalized by the predictions at NLO accuracy.

numerically because the choice of the fixed scales results in a badly converging perturbation series. We found the same feature for any other distribution.

In Ref. [8] the dynamical scale $\mu_{0}^{2}=m_{\mathrm{t}} \sqrt{p_{\mathrm{b} \perp} p_{\overline{\mathrm{b}} \perp}}$ was found more appropiate for this process, leading to smaller NLO corrections, indicating better convergence of the perturbation series. Unfortunately, this scale cannot be implemented in our computations. Instead, we made predictions with another dynamical scale, related to sum of the transverse mass $m_{f, \perp}=\sqrt{m_{f, \perp}^{2}+p_{f, \perp}^{2}}$ of the particles in the final state,

$$
H_{\mathrm{T}}=\sum_{f} m_{f, \perp}
$$

by $\mu_{0}=H_{\mathrm{T}} / 4$. In a leading order computation this is the arithmetic mean of the transverse masses of the final state particles. Indeed, we also found that this dynamical scale leads to much smaller NLO corrections and as a result, much better agreement between the distributions from the LHE's and those at NLO accuracy.

We compare our predictions from the LHE's with this default scale to the NLO prediction of Ref. [8] in Fig. 2. We observe remarkable agreement between these two predictions. We found similar agreement for other distributions presented in Ref. [8]. On the same figure also show the scale dependence as shaded band when the equal renormalization and factorization scales are varied in the range $\left[\mu_{0} / 2,2 \mu_{0}\right]$. We adopt the dynamical scale $\mu_{0}=H_{\mathrm{T}} / 4$ in our further analysis when we make predictions at the hadron level.

Feeding the LHE's into the SMC's enables us to make predictions not only at the hadron level. In addition to the distributions discussed above (at (i) NLO accuracy, and (ii) from the pre-showered POWHEG simulation referred to LHE's, formally at the NLO accuracy), we can make predictions (iii) after decay of the heavy particles, and (iv) at the hadron level after full SMC. For experimental analyses the last option is the most useful. For examining the effect of the different stages in the evolution, it is useful to study the predictions at intermediate stages. 

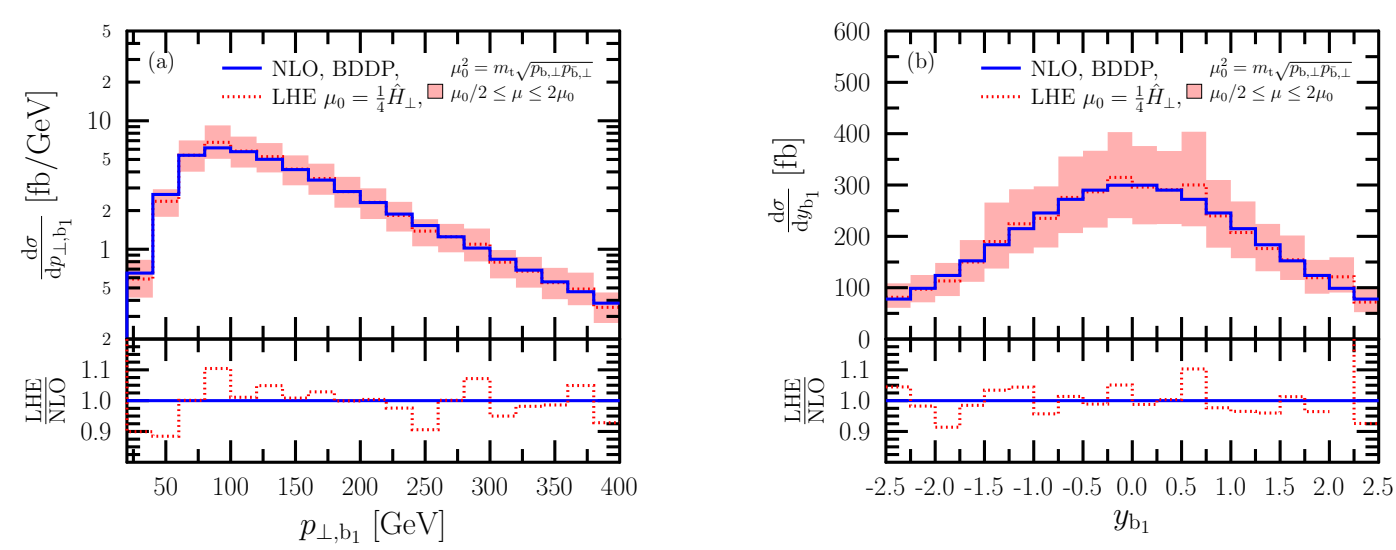

Figure 2: Same as Fig. 1, but with dynamical scales as shown in the legend.

We use PYTHIA-6.4.26 [23] for studying the effect of the shower and hadronization. We set the masses of heavy bosons in PYTHIA to be the same as in PowHel. We switched off the contribution of photon emission from leptons, multiple interactions and enforced B-hadron stability. All other particles and hadrons were assumed to be stable or decay according to the PYTHIA default implementation.

We have generated pre-showered events at collider energy $\sqrt{s}=8 \mathrm{TeV}$, with t-quarks of mass $m_{\mathrm{t}}=173.2 \mathrm{GeV}$, and at equal renormalization and factorization scales $\mu_{\mathrm{R}}=\mu_{\mathrm{F}}=\mu_{0}=H_{\mathrm{T}} / 4$. Of course, the SMC changes the particle content significantly, and thus the effect of selection cuts, too. In order to compare distributions from the same set of events, we select LHE's similarly as above. First we clustered tracks into jets with the anti- $k_{\perp}$-algorithm (as implemented in Fast Jet Ref. [22]) using $R=0.4$. A track was considered a possible jet constituent if $\left|\eta^{\text {track }}\right|<5$. We excluded t-quarks from the set of possible tracks. Then we employed the following selection cuts for these distributions:

1. events with invariant mass of the $b \bar{b}$-jet pair below $m_{b \bar{b}}^{\min }=100 \mathrm{GeV}$ were discarded,

2. $p_{\perp, j}^{\min }=25 \mathrm{GeV}$,

3. we required at least two jets, one $b$ - and one $\bar{b}$-jet, with $\left|\eta_{b(\bar{b})}\right|<2.5$.

Then we let the heavy particles decay and produced distributions at this stage. We compare those to distributions obtained from the same set of events after full SMC, employing the cuts $p_{\perp, j}^{\min }=$ $25 \mathrm{GeV}$, and $\left|\eta_{j}\right|<2.5$ also on the jets clustered from particles available at the two stages, using the anti- $k_{\perp}$-algorithm with $R=0.4$. Such comparison is shown in Fig. 3 for the hardest and second hardest $b \bar{b}$-jet pairs for the distributions of the invariant masses and separation in pseudorapidityazimuthal angle plane. We see that the effect of SMC is small on the hardest pairs that more likely originate in the hard scattering. In fact it is negligible for the $\Delta R_{b \bar{b}}$-separation and also for the invariant mass in the region $[100,150] \mathrm{GeV}$, interesting for Higgs studies. For other jet pairs however, the effect of the shower can be larger. It is fairly uniform decrease of about $25 \%$ in the $\Delta R_{b \bar{b}}$-separation, and a significant softening in the invariant mass distribution. This feature is quite general: the effect of the SMC is small on angular variables, but there is a sizeable softening of transverse momentum distributions. 

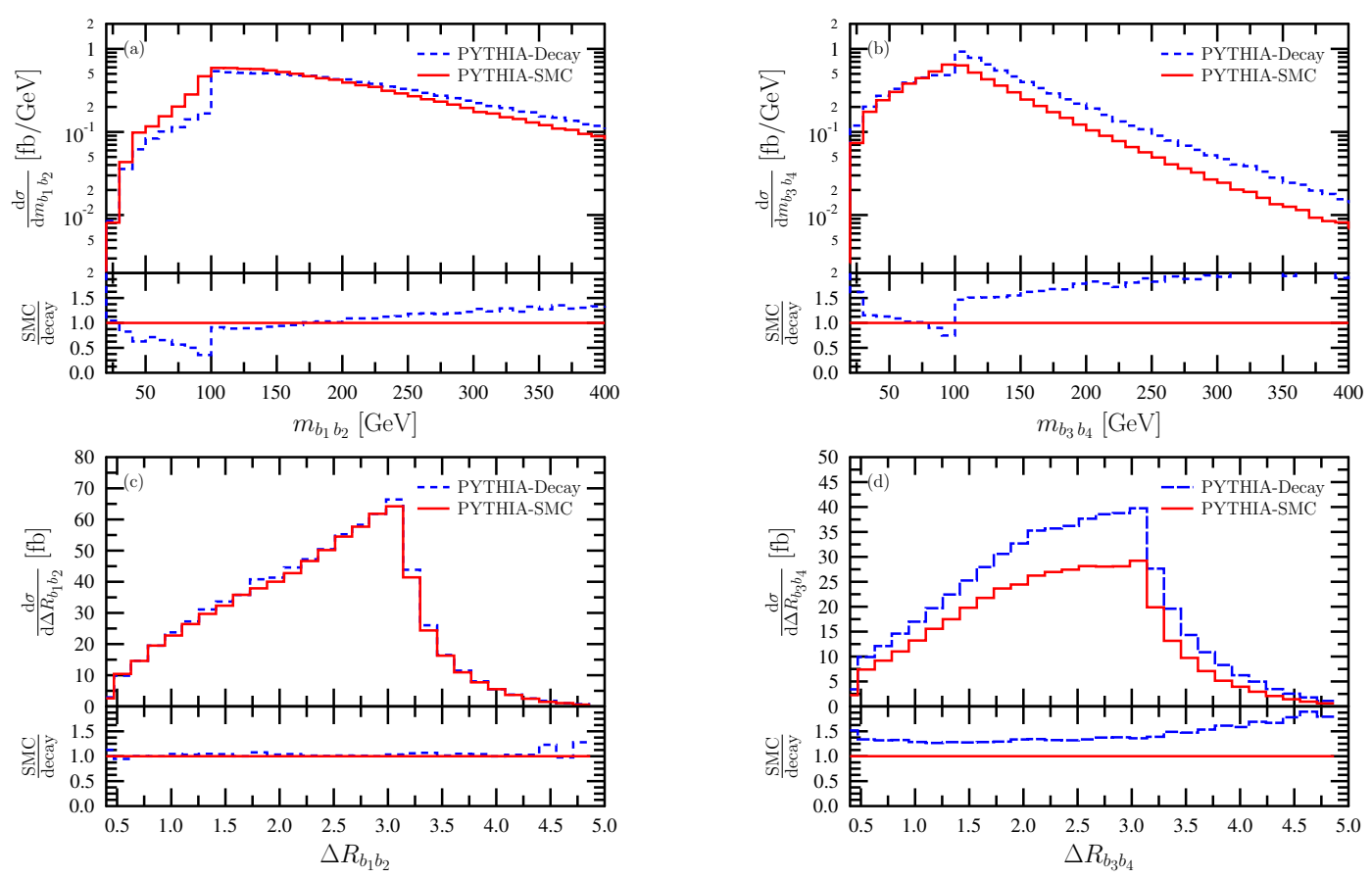

Figure 3: Kinematic distributions of the $b \bar{b}$-jet pairs after decay and after full SMC: (a) invariant mass of the two hardest $b \bar{b}$ jets, (b) invariant mass of the two second hardest $b \bar{b}$ jets, (c) $\Delta R$-separation of the two hardest $b \bar{b}$ jets, (d) $\Delta R$-separation of the two second hardest $b \bar{b}$ jets. The lower panels show the SMC predictions normalized by the predictions from events after decay.
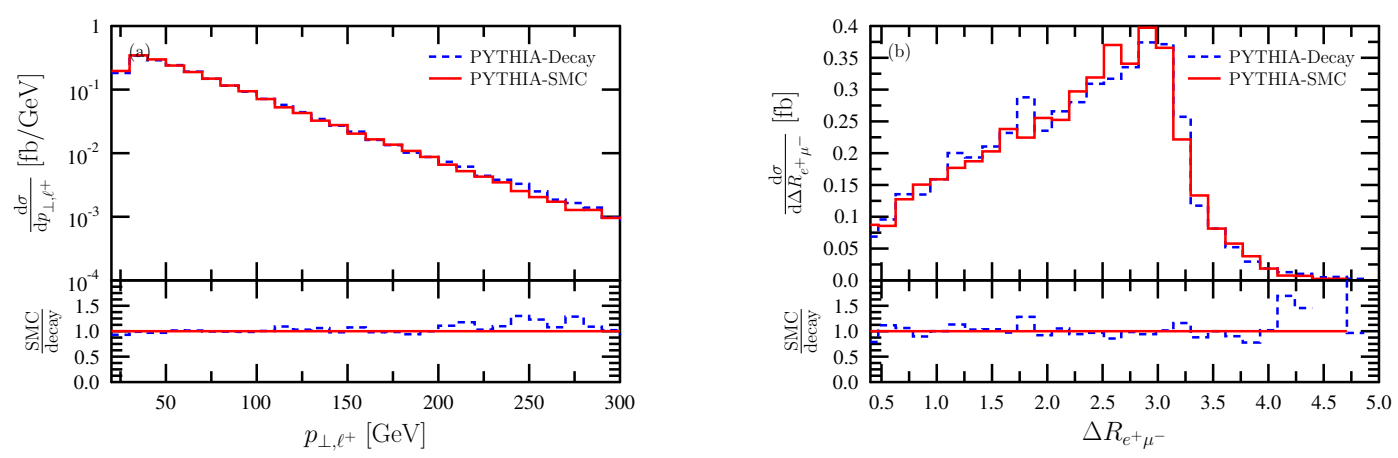

Figure 4: Kinematic distributions of the (a) hardest positively charged isolated lepton and (b) $\Delta R$-separation of the hardest isolated positron and muon after decay and after full SMC. The lower panels show the SMC predictions normalized by the predictions from events after decay.

With this tool one can also study kinematic distributions of leptons. In Fig. 4.a we show the distribution of the transverse momentum of the positively charged isolated leptons and in Fig. 4.b the distribution of the separation between the hardest isolated positron and muon in the pseudorapidityazimuthal angle. For these (and other) distibutions of leptons the effect of the shower is small as expected. (However, the selection cuts if applied on hadrons instead of LHE's can change the cross sections significantly.)

This is a part of a bigger project with the aim of providing event files produced by PowHel 
for processes $p p \rightarrow \mathfrak{t} \mathfrak{t}+X$, where $X$ is a hard object (SM boson, jet, b-quark pair). These files are available at http://grid.kfki.hu/twiki/bin/view/DbTheory/ together with the corresponding version of the program. Using those events an analysis for optimizing the selection for $t \bar{t}+H$-events is in progress.

Note added: After the conference a paper [24] on very similar topic appeared.

\section{References}

[1] Aad G. et al., Phys.Lett. B, 710, 49 (2012).

[2] Chatrchyan S. et al., Phys.Lett. B, 710, 26 (2012).

[3] Beringer J. et al., Phys.Rev. D86, 010001 (2012).

[4] Maltoni F., Rainwater D. L. and Willenbrock S., Phys.Rev. D, 66, 034022 (2002).

[5] Belyaev A. and Reina L., JHEP, 0208, 041 (2002).

[6] Bredenstein, A. Denner A., Dittmaier S. and Pozzorini S., Phys. Rev. Lett. 103, 012002 (2009). [arXiv:0905.0110 [hep-ph]].

[7] Bevilacqua G., Czakon M., Papadopoulos C. G., Pittau R. and Worek M., JHEP 0909, 109 (2009).

[8] Bredenstein A., Denner A., Dittmaier S. and Pozzorini S., JHEP 1003, 021 (2010).

[9] P. Nason, JHEP 0411, 040 (2004) [arXiv:hep-ph/0409146].

[10] S. Frixione, P. Nason and C. Oleari, JHEP 0711, 070 (2007) [arXiv:0709.2092].

[11] S. Alioli, P. Nason, C. Oleari and E. Re, JHEP 1006, 043 (2010) [arXiv:1002.2581].

[12] M. V. Garzelli, A. Kardos and Z. Trocsanyi, PoS EPS -HEP2011, 282 (2011) [arXiv:1111.1446 [hep-ph]].

[13] G. Bevilacqua et al., arXiv:1110.1499.

[14] J. Alwall et al., Comput. Phys. Commun. 176, 300 (2007) [arXiv:hep-ph/0609017].

[15] A. Kardos, C. Papadopoulos and Z. Trocsanyi, Phys. Lett. B 705, 76 (2011) [arXiv:1101.2672 [hep-ph]].

[16] M. V. Garzelli, A. Kardos, C. G. Papadopoulos and Z. Trocsanyi, Europhys. Lett. 96, 11001 (2011) [arXiv:1108.0387 [hep-ph]].

[17] M. V. Garzelli, A. Kardos, C. G. Papadopoulos and Z. Trocsanyi, Phys. Rev. D 85, 074022 (2012) [arXiv:1111.1444 [hep-ph]].

[18] M. V. Garzelli, A. Kardos and Z. Trocsanyi, PoS LL 2012, 057 (2012).

[19] M. V. Garzelli, A. Kardos, C. G. Papadopoulos and Z. Trocsanyi, JHEP 1211, 056 (2012) [arXiv:1208.2665 [hep-ph]].

[20] M. V. Garzelli, A. Kardos, C. G. Papadopoulos and Z. Trocsanyi, arXiv:1302.5381 [hep-ph].

[21] A. Kardos and Z. Trocsanyi, arXiv:1303.6291 [hep-ph].

[22] M. Cacciari, G. P. Salam and G. Soyez, Eur. Phys. J. C 72, 1896 (2012) [arXiv:1111.6097 [hep-ph]], http://fastjet.fr

[23] T. Sjostrand, S. Mrenna and P. Z. Skands, JHEP 0605, 026 (2006) [arXiv:hep-ph/0603175].

[24] F. Cascioli, P. Maierhoefer, N. Moretti, S. Pozzorini and F. Siegert, arXiv:1309.5912 [hep-ph]. 